\title{
A COMPLETENESS THEOREM FOR TRIGONOMETRIC IDENTITIES AND VARIOUS RESULTS ON EXPONENTIAL FUNCTIONS
}

\author{
LOU VAN DEN DRIES ${ }^{1}$
}

\begin{abstract}
All valid identities in terms of variables, real constants, the arithmetic operations of addition and multiplication, and the trigonometric operations of sine and cosine are shown to be consequences of a few familiar identities and numerical facts. We also indicate how to decide whether $f$ eventually dominates $g$, for $f$ and $g$ from a certain class of exponential functions. Finally, we correct a statement from an earlier paper.
\end{abstract}

Introduction. Consider the familiar identities:

(1) $\cos (x+y)=\cos x \cdot \cos y-\sin x \cdot \sin y$,

(2) $\sin (x+y)=\cos x \cdot \sin y+\sin x \cdot \cos y$,

(3) $\cos (-x)=\cos x, \sin (-x)=-\sin x$.

From these identities and numerical facts like $\cos 0=1$ we can derive other identities like $\cos ^{2} x+\sin ^{2} x=1$. The question came up (cf. [HR, p. 31]) whether all valid identities formulated in terms of variables, individual real numbers, the functions $\cos$ and $\sin$, and the ring operations,,$+- \cdot$ can be derived from the identities (1)-(3) above, plus the identities defining commutative rings with unit 1 , plus the "true numerical facts", these being the valid identities not containing variables. (We allow terms like $\sin (\cos x)$.)

In $\S 1$ we shall answer this question affirmatively. ${ }^{2}$

In [L, p. 219] H. Levitz asked whether "eventual dominance" for a certain class of exponential functions could be effectively decided. We now indicate a larger class of exponential functions for which we found a decision method. To describe this class of functions let us define a Skolem monomial to be a formal product

$$
M=P_{0} \cdot P_{1}^{Q_{1}} \ldots P_{k}^{Q_{k}}
$$

where $P_{0}, P_{1}, \ldots, P_{k}$ are rational functions in $X$ over $\mathbf{Q}$, such that $P_{1}(x), \ldots, P_{k}(x)$ are $>0$ for all sufficiently large real $x$, and $Q_{1}, \ldots, Q_{k}$ are polynomials in $X$ over Q. (So $M(x)$ is a well-defined real number for sufficiently large $x$.)

If $M_{1}, \ldots, M_{s}$ are Skolem monomials, then either $M_{1}(x)+\cdots+M_{s}(x)>0$ for all sufficiently large $x$, or $M_{1}(x)+\cdots+M_{s}(x)=0$ for all sufficiently large $x$, or $M_{1}(x)+\cdots+M_{s}(x)<0$ for all sufficiently large $x$.

This fact follows from the main theorem in Hardy's book $[\mathbf{H}]$, but the proof there does not lead to a decision procedure.

Received by the editors January 21, 1985

1980 Mathematics Subject Classification. Primary 03B25, 03D40, 26A09, 26A12; Secondary 03C05, 08A50, 08B05.

${ }^{1}$ Supported by the NSF.

${ }^{2}$ In a discussion with $\mathrm{W}$. Henson we found that the addition law for the cosine and the symmetry law for the $\operatorname{cosine}(\cos (-x)=\cos x)$ are derivable from the other identities, but that one cannot also leave out $\sin (-x)=-\sin x$, as was inadvertently done in [HR, Corollary 5.3]. 
In $\S 2$ we show how to decide for any given Skolem monomials $M_{1}, \ldots, M_{s}$ which of the three alternatives holds. The proof depends on unique factorization and the fact that $e$ is transcendental.

In $\S 3$ we point out a false statement in the author's article [VdD], and substitute a correct statement. I thank E. Bouscaren for casting doubt on the statement in [VdD].

1. A completeness theorem for trigonometric identities.

(1.1) Let $L=\{0,1,+,-, \cdot\}$ be the usual language of rings with unit 1 and $L_{\mathbf{R}}=L$ augmented by a constant symbol (name) for each real number. Further let $c$ and $s$ be two extra 1-place function symbols and define $\operatorname{Tr}(\mathbf{R})$ (for trigonometry over $\mathbf{R}$ ) to be the equational theory in the language $L_{\mathbf{R}} \cup\{c, s\}$ axiomatized by the following identities:

(1) the $L$-identities defining commutative rings with 1 ;

(2) the trigonometric identities: $c(x+y)=c(x) c(y)-s(x) s(y), s(x+y)=$ $c(x) s(y)+s(x) c(y), c(-x)=c(x), s(-x)=-s(x)$

(3) the true numerical facts: all sentences $\tau=0$ true in $\mathbf{R}$, where $\tau$ is an $L_{\mathbf{R}} \cup\{c, s\}$-term not containing variables; the symbols $c$ and $s$ are to be interpreted as $\cos$ and $\sin$ respectively.

(1.2) THEOREM. Let $\tau(\bar{x}), \bar{x}=\left(x_{1}, \ldots, x_{n}\right)$, be an $L_{\mathbf{R}} \cup\{c, s\}$-term such that $\tau(\bar{r})=0$ for each $\bar{r}=\left(r_{1}, \ldots, r_{n}\right) \in \mathbf{R}^{n}$. Then $\operatorname{Tr}(\mathbf{R}) \vdash \forall \bar{x}(\tau(\bar{x})=0)$.

(1.3) The proof is somewhat indirect, and we first derive a completeness result for identities which may also involve the exponential function $\exp (x)=e^{x}$.

So let $e$ be a new 1-place function symbol and define $T_{(e, c, s)}$ as the equational theory in the language $L_{(e, c, s)}=L \cup\{1 / 2, e, c, s\}$ axiomatized by:

(a) the identities for commutative rings with 1 , plus the identity $\frac{1}{2}+\frac{1}{2}=1$,

(b) $e(x+y)=e(x) \cdot e(y), e(0)=1$,

(c) the trigonometric identities as in (2) above,

(d) all sentences $\tau=0$ which are true on $\mathbf{R}$, where $\tau$ is an $L_{(e, c, s)}$-term not containing variables. (Here $e, c, s$ are interpreted as exp, $\cos$ and $\sin$ on $\mathbf{R}$.)

(1.4) THEOREM. Let $\tau(\bar{x}), \bar{x}=\left(x_{1}, \ldots, x_{n}\right)$, be an $L_{(e, c, s)}$-term such that $\tau(\bar{r})=0$ for all $\bar{r}=\left(r_{1}, \ldots, r_{n}\right) \in \mathbf{R}^{n}$. Then

$$
T_{(e, c, s)} \vdash \forall \bar{x}(\tau(\bar{x})=0) .
$$

(1.5) First we have a simple lemma. Let $i$ be a new constant symbol and define Euler as the equational $L_{e, c, s}(i)$-theory axiomatized by Euler's identities

$$
c(x)=(1 / 2) \cdot(e(i x)+e(-i x)), \quad s(x)=(-1 / 2) i \cdot(e(i x)-e(-i x)) .
$$

These identities can be used to eliminate the symbols $c$ and $s$ at the expense of introducing the new symbol $i$, in other words, we have

(1.6) LEMMA. For each $L_{(e, c, s)}$-term $\tau(\bar{x}), \bar{x}=\left(x_{1}, \ldots, x_{n}\right)$, there is an $L \cup$ $\{1 / 2, i, e\}$-term $\tau_{e}(\bar{x})$ such that Euler $\vdash \tau(\bar{x})=\tau_{e}(\bar{x})$.

(1.7) ProOF OF THEOREM (1.4). By model theory it suffices to show that, given any model $\underline{R}$ of $T_{(e, c, s)}$, we have $\underline{R} \vDash \forall \bar{x}(\tau(\bar{x})=0)$. Let $A$ be the smallest subset of $\mathbf{R}$ containing $0,1 / 2,1$ and closed under $+,-, \cdot, \exp , \cos$ and sin. Clearly 
$A$ is the underlying set of a $T_{(e, c, s)}$-model $\underline{A}$. Let $\Phi: \underline{A} \rightarrow \underline{R}$ be the unique $L_{(e, c, s)^{-}}$ morphism. We consider $\underline{R}$ as an $\underline{A}$-algebra via $\Phi$. The subring $\underline{A}[i]=\underline{A}+\underline{A} \cdot i$ of $\mathbf{C}$ is also closed under exp, $\cos$ and sin, since $\exp (a+b i)=\exp a \cdot(\cos b+i \sin b)$.

Consider now the $\underline{A}[i]$-algebra $\underline{R} \otimes_{\underline{A}} \underline{A}[i]$. The usual identifications give that $\underline{R} \otimes_{A} \underline{A}[i]=\underline{R}[i]$, and $\underline{R}[i]$ is a free $\underline{R}$-module on the basis $1, i$. This allows us to extend the operations $e, c, s$ on $\underline{R}$ to $\underline{R}[i]:$ put $e(\lambda+\mu i)=e(\lambda) \cdot(c(\mu)+i \cdot s(\mu))$ for $\lambda, \mu \in \underline{R}$. It is easy to verify that the operation $e$ on $\underline{R}[i]$ extends the operation $e$ on $\underline{R}$ and is a morphism of the additive group of $\underline{R}[i]$ into the multiplicative group of units of $\underline{R}[i]$. We also put, for $z \in \underline{R}[i]$ :

$$
c(z)=(1 / 2) \cdot(e(i z)+e(-i z)), \quad s(z)=(-1 / 2) i \cdot(e(i z)-e(-i z)),
$$

and again $c$ and $s$ on $\underline{R}[i]$ extend $c$ and $s$ on $\underline{R}$. It is clear that the structural morphism $\underline{A}[i] \rightarrow \underline{R}[i]$ is also a morphism for the $e$-operation. From the assumption that $\tau(\bar{r})=0$ for all $\bar{r} \in \mathbf{R}^{n}$ we get by analytic continuation that $\tau(\bar{r})=0$ for all $\bar{r} \in \mathbf{C}^{n}$. Let $\tau_{e}(\bar{x})$ be an $L \cup\{1 / 2, i, e\}$-term such that Euler $\vdash \tau(\bar{x})=\tau_{e}(\bar{x})$. (Such a term exists by the lemma.) Hence $\underline{A}[i] \vDash \forall \bar{x}\left(\tau_{e}(\bar{x})=0\right)$, and since the $E$-ring $\underline{A}[i]$ satisfies the hypotheses of [VdD, Proposition (4.1)] (with $\left.r=1, d_{i}=\partial / \partial x_{i}\right)$, we get that $\forall \bar{x}\left(\tau_{e}(\bar{x})=0\right)$ is also true for every $E$-ring $S$ for which there exists an $E$-ring morphism $\underline{A}[i] \rightarrow S$ (where $1 / 2, i$ are to be interpreted by their image in $S$ under this morphism). In particular the sentence is true in $S=\underline{R}[i]$, and since $\underline{R}[i] \vDash$ Euler, we get $\underline{R}[i] \vDash \forall \bar{x}(\tau(\bar{x})=0)$, hence $\underline{R} \vDash \forall \bar{x}(\tau(\bar{x})=0)$.

(1.8) For the proof of Theorem (1.2) we need the following relativization of (1.4). Let $L_{(e, c, s)}(\mathbf{R})$ be the language $L_{(e, c, s)}$ augmented by a constant symbol for each real number, and let $T_{(e, c, s)}(\mathbf{R})$ be the equational $L_{(e, c, s)}(\mathbf{R})$-theory which has the same axioms as $T_{(e, c, s)}$ except that in clause (d) we allow $L_{(e, c, s)}(\mathbf{R})$-terms (see (1.3)).

We now have the following result which is proved in the same way as Theorem (1.4).

Let $\tau(\bar{x}), \bar{x}=\left(x_{1}, \ldots, x_{n}\right)$, be an $L_{(e, c, s)}(\mathbf{R})$-term such that $\tau(\bar{r})=0$ for all $\bar{r} \in \mathbf{R}^{n}$. Then $T_{(e, c, s)}(\mathbf{R}) \vdash \forall \bar{x}(\tau(\bar{x})=0)$.

(1.9) We can now prove our trigonometric completeness result.

PROOF OF THEOREM (1.2). By model theory it suffices to show that, given any model $R$ of $\operatorname{Tr}(\mathbf{R})$, we have $R \vDash \forall \bar{x}(\tau(\bar{x})=0)$. We may of course assume $R \neq\{0\}$. We have a canonical $L_{\mathbf{R}} \cup\{c, s\}$-morphism $\Phi: \mathbf{R} \rightarrow R$. Since $\mathbf{R}$ is a field and $R \neq\{0\}, \Phi$ is injective and $\Phi(\mathbf{R})$ is a direct summand of the $\mathbf{R}$ linear space $R$, say $R=\Phi(\mathbf{R}) \oplus B$. We equip $R$ with an operation $e$ by putting $e(\Phi(r)+b)=\Phi(\exp (r))(r \in \mathbf{R}, b \in B)$. It is easy to see that then $(R, e)$ is an $E$-ring and that $\Phi$ is also an $E$-ring morphism $(\mathbf{R}, \exp ) \rightarrow(R, e)$. In other words: $(R, e) \vDash T_{(e, c, s)}(\mathbf{R})$ (see $(1.8)$ ), whence $(R, e) \vDash \forall \bar{x}(\tau(\bar{x})=0)$. But the symbol $e$ does not occur in $\tau$, therefore $R \vDash \forall \bar{x}(\tau(\bar{x})=0)$.

(1.10) Negative results on identity problems for elementary functions can be found in $[\mathbf{R 1}]$.

\section{A decision method for eventual dominance.}

(2.1) In this section we solve a slightly generalized form of a decision problem posed by H. Levitz (see the Introduction). Another solution of Levitz's problem was found by R. Gurevic [G]. 
As is common in dealing with effectivity notions we abuse language to avoid unwieldy formulations. For instance, we assume tacitly that rings like $\mathbf{Q}$ and $\mathbf{Q}[X]$ are recursively presented. To give a concrete example, the precise formulation of Lemma (2.2) below is as follows:

There is an algorithm which, for any polynomials $f(X), g(X) \in \mathbf{Q}[X]$ with $g(X) \neq 0$, computes a triple $(a, i, e) \in \mathbf{Q} \times \mathbf{Z} \times \mathbf{N}$ such that

$$
f(X) / g(X)=a X^{i} \cdot\left(1+a_{1} X^{-1}+a_{2} X^{-2}+\cdots\right)
$$

in the formal power series field $\mathbf{Q}\left(\left(X^{-1}\right)\right)$, where $a_{1}, a_{2}, a_{3}, \ldots$ is a recursive sequence of rationals with index $e$.

In the sequel we shall leave the translation of our statements into such pedantically precise language to the reader.

(2.2) LEMMA. Given a rational function $P(X) \in \mathbf{Q}(X)$ one can compute a rational number $a$, an integer $i$, and a sequence of rationals $a_{1}, a_{2}, a_{3}, \ldots$, such that

$$
P(X)=a X^{i} \cdot\left(1+a_{1} X^{-1}+a_{2} X^{-2}+\cdots\right) .
$$

PROOF. If $P$ is a polynomial $a_{n} X^{n}+a_{n-1} X^{n-1}+\cdots+a_{0}$ with $a_{n} \neq 0$, then

$$
P=a_{n} X^{n} \cdot\left(1+\frac{a_{n-1}}{a_{n}} X^{-1}+\cdots+\frac{a_{0}}{a_{n}} X^{-n}+0\right)
$$

also

$$
\frac{1}{P}=a_{n}^{-1} X^{-n} \cdot\left(1-\left(\frac{a_{n-1}}{a_{n}} X^{-1}+\cdots+\frac{a_{0}}{a_{n}} X^{-n}\right)+(\cdots)^{2} \cdots\right),
$$

and one computes easily successive rationals $b_{1}, b_{2}, b_{3}, \ldots$ such that this can be written as

$$
a_{n}^{-1} X^{-n} \cdot\left(1+b_{1} X^{-1}+b_{2} X^{-2}+\cdots\right) \text {. }
$$

The case of a quotient of polynomials can be reduced to the above cases and multiplication of power series in $X^{-1}$ with given rational coefficients.

(2.3) LEMMA. Given (the index of) a recursive sequence of rationals $a_{1}, a_{2}, a_{3}$, ... one can compute a sequence of rationals $b_{1}, b_{2}, b_{3}, \ldots$ such that

$$
\log \left(1+a_{1} X^{-1}+a_{2} X^{-2}+\cdots\right)=b_{1} X^{-1}+b_{2} X^{-2}+b_{3} X^{-3}+\cdots
$$

PROOF. The formal power series $\log (1+Y)$ is defined by: $\log (1+Y)=Y-$ $Y^{2} / 2+Y^{3} / 3-\cdots$, and the result follows by substitution.

(2.4) In the next lemmas we use expressions $E(X)$ containing the indeterminate $X$ such that $E(x)$ is a well-defined real number for all sufficiently large $x$. Such an expression stands for the germ at $\infty$ of the real function it defines. So an identity $E_{1}(X)=E_{2}(X)$ means that $E_{1}(x)=E_{2}(x)$ for all $x>r$, for some $r \in \mathbf{R}$ such that $E_{1}(x)$ and $E_{2}(x)$ are defined for $x>r$.

For $P(X) \in \mathbf{Q}(X)$ we write $P(X)>0$ if $P(x)>0$ for all sufficiently large $x$. So $P(X)>0$ if and only if $a>0$, where $a$ is as in Lemma (2.2).

Finally, we let $p_{1}=2, p_{2}=3, \ldots, p_{n}, \ldots$ be the successive prime numbers.

(2.5) LemMA. Given $P(X) \in \mathbf{Q}(X)$ and $Q(X) \in \mathbf{Q}[X]$, with $P(X)>0$, one can compute a positive integer $n$ and polynomials $q(X), q_{1}(X), \ldots, q_{n}(X), q_{\infty}(X) \in$ $\mathbf{Q}[X]$, and a sequence of rationals $c_{1}, c_{2}, c_{3}, \ldots$, such that

$$
P(X)^{Q(X)}=e^{q(X)} \cdot 2^{q_{1}(X)} \cdots \cdots p_{n}^{q_{n}(X)} \cdot X^{q_{\infty}(X)} \cdot\left(1+c_{1} X^{-1}+c_{2} X^{-2}+\cdots\right) .
$$


ProOF. Write $P(X)=a X^{i} \cdot\left(1+a_{1} X^{-1}+a_{2} X^{-2}+\cdots\right)$ as in (2.2). Then

$$
\begin{aligned}
P(X)^{Q(X)} & =e^{Q(X) \cdot \log P(X)} \\
& =e^{\left.Q(X) \cdot\left[\log a+i \log X+\log \left(1+a_{1} X^{-1}+\cdots\right)\right] \quad \text { (using }(2.3)\right)} \\
& =e^{Q(X) \cdot(\log a+i \log X)+Q(X) \cdot\left[b_{1} X^{-1}+b_{2} X^{-2}+\cdots\right]} \\
& =e^{q(X)+q_{l}(X) \log a+q_{\infty}(X) \log X} \cdot e^{d_{1} X^{-1}+d_{2} X^{-2}+\cdots},
\end{aligned}
$$

where $q(X), q_{l}(X), q_{\infty}(X) \in \mathbf{Q}[X]$, and the sequence of rationals $d_{1}, d_{2}, d_{3}, \ldots$ can be computed from $Q(X), i$, and the sequence $b_{1}, b_{2}, b_{3}, \ldots$

Now factor $a$ as $a=2^{k_{1}} \cdot 3^{k_{2}} \cdots \cdots p_{n}^{k_{n}}\left(k_{i} \in \mathbf{Z}\right)$, so we obtain $q_{l}(X) \log a=$ $q_{1}(X) \log 2+q_{2}(X) \log 3+\cdots+q_{n}(X) \log p_{n}$ for polynomials $q_{1}, \ldots, q_{n} \in \mathbf{Q}[X]$. Furthermore, substituting $d_{1} X^{-1}+d_{2} X^{-2}+\cdots$ in the power series $e^{Y}=1+Y+$ $Y^{2} / 2 !+\cdots$, we can compute rationals $c_{1}, c_{2}, c_{3}, \ldots$, such that

$$
e^{d_{1} X^{-1}+d_{2} X^{-1}+\cdots}=1+c_{1} X^{-1}+c_{2} X^{-2}+\cdots
$$

The result is

$$
P(X)^{Q(X)}=e^{q(X)} \cdot 2^{q_{1}(X)} \cdots \cdots p_{n}^{q_{n}(X)} \cdot X^{q_{\infty}(X)} \cdot\left(1+c_{1} X^{-1}+c_{2} X^{-1}+\cdots\right) .
$$

(2.6) LemMa. Given $P_{1}(X)>0, \ldots, P_{k}(X)>0$ in $\mathbf{Q}(X)$ and polynomials $Q_{1}(X), \ldots, Q_{k}(X) \in \mathbf{Q}[X]$ one can compute a positive integer $n$, polynomials $q(X), q_{1}(X), \ldots, q_{n}(X), q_{\infty}(X) \in \mathbf{Q}[X]$, and a sequence of rationals $c_{1}, c_{2}, c_{3}, \ldots$, such that

$$
\begin{aligned}
& P_{1}(X)^{Q_{1}(X)} \cdot P_{2}(X)^{Q_{2}(X)} \cdots P_{k}(X)^{Q_{k}(X)} \\
& \quad=e^{q(X)} \cdot 2^{q_{1}(X)} \cdot 3^{q_{2}(X)} \cdots \cdots p_{n}^{q_{n}(X)} \cdot X^{q_{\infty}(X)} \cdot\left(1+c_{1} X^{-1}+c_{2} X^{-2}+\cdots\right) .
\end{aligned}
$$

Proof. The proof is immediate from Lemma (2.5). Note that one can take the same $n$ for all powers $P_{i}(X)^{Q_{i}(X)}$ by letting some exponents be equal to zero, if necessary.

(2.7) LEMMA. If $r, r_{1}, \ldots, r_{n} \in \mathbf{Q}$, then $r+r_{1} \log 2+r_{2} \log 3+\cdots+r_{n} \log p_{n}=0$ if and only if $r=r_{1}=\cdots=r_{n}=0$. For given $r, r_{1}, \ldots, r_{n} \in \mathbf{Q}$ one can determine effectively which of the three statements

$$
r+r_{1} \log 2+\cdots+r_{n} \log p_{n}\left\{\begin{array}{l}
=0 \\
>0 \\
<0
\end{array}\right.
$$

holds.

PROOF. We may assume the $r_{i}$ are integers. Then $r+r_{1} \log 2+\cdots+r_{n} \log p_{n}=$ $0 \Leftrightarrow e^{r}=2^{-r_{1}} \cdots \cdots p_{n}^{-r_{n}}$. Since $e$ is transcendental, this can only happen when $r=0$, which, by unique factorization, implies $r_{1}=\cdots=r_{n}=0$. The second statement follows from the first and from the fact that we can compute rational approximations to $\log p$ for $p$ a given positive integer.

(2.8) In the following we consider tuples $\sigma=\left(q, q_{1}, \ldots, q_{n}, q_{\infty}\right)(n \geq 0)$ with each term in $X \cdot \mathbf{Q}[X]$, i.e., $q, q_{i}(1 \leq i \leq n)$ and $q_{\infty}$ are polynomials in $X$ over $\mathbf{Q}$ with constant term 0 .

To such a tuple $\sigma$ we associate the formal product $F_{\sigma}=e^{q} \cdot 2^{q_{1}} \cdots \cdot p_{n}^{q_{n}} \cdot X^{q_{\infty}}$.

Note that, given any two tuples $\sigma_{1}$ and $\sigma_{2}$, one can determine a tuple $\tau$ such that $F_{\sigma_{1}}(x) / F_{\sigma_{2}}(x)=F_{\tau}(x)$ for all $x>0$; by inserting dummy zeros if necessary 
we may assume that $\sigma_{1}$ and $\sigma_{2}$ are of the same length and then one obtains $\tau$ by $\tau=\sigma_{1}-\sigma_{2}$.

(2.9) LEMMA. For any tuple $\sigma \neq(0,0, \ldots, 0)$ there is a constant $c>0$ such that either $F_{\sigma}(x)<e^{-c x}$ for all sufficiently large $x$, or $F_{\sigma}(x)>e^{c x}$ for all sufficiently large $x$.

For any given tuple $\sigma \neq(0,0, \ldots, 0)$ one can determine effectively which of the two cases takes place.

ProOF. Let $\sigma=\left(q, q_{1}, \ldots, q_{n}, q_{\infty}\right)$, and let $k$ be the maximum of the degrees of the terms of $\sigma$. Then we can write

$$
F_{\sigma}=\exp \left(\sum_{i=1}^{k} X^{i}\left(r_{i}+r_{i_{1}} \log 2+\cdots+r_{i_{n}} \log p_{n}+r_{i_{\infty}} \log X\right)\right) .
$$

Here the $r_{i}, r_{i_{j}}$ and $r_{i_{\infty}}$ are rational numbers which we can compute from $\sigma$. By definition of $k$ one of the numbers $r_{k}, r_{k_{j}}, r_{k_{\infty}}$ is not zero. If $r_{k_{\infty}}<0$, then $F_{\sigma}(x)<e^{-c x}$ for some $c>0$ and all sufficiently large $x$.

Suppose $r_{k_{\infty}}=0$. By Lemma (2.7) either $r_{k}+r_{k_{1}} \log 2+\cdots+r_{k_{n}} \log p_{n}<0$ or $r_{k}+r_{k_{1}} \log 2+\cdots+r_{r_{n}} \log p_{n}>0$. In the first case we have again $F_{\sigma}(x)<e^{-c x}$ for some $c>0$ and all sufficiently large $x$, and in the second case $F_{\sigma}(x)>e^{c x}$ for some $c>0$ and all sufficiently large $x$.

(2.10) Lemma. Given $P_{0}(X) \in \mathbf{Q}(X), P_{1}(X)>0, \ldots, P_{k}(X)>0$ in $\mathbf{Q}(X)$ and polynomials $Q_{1}(X), \ldots, Q_{k}(X) \in \mathbf{Q}[X]$ we can effectively determine a tuple $\sigma$, positive integers $m$ and $M$, a rational number $r$, and a sequence of algebraic real numbers $d_{-m}, d_{-m+1}, \ldots, d_{0}, d_{1}, d_{2}, \ldots$ such that, with $f_{i}=d_{i} \cdot e^{r}$, we have

$$
\begin{aligned}
P_{0} \cdot & P_{1}^{Q_{1}} \cdots P_{k}^{Q_{k}} \\
& =F_{\sigma} \cdot\left(f_{-m} X^{m / M}+f_{-m+1} X^{(m-1) / M}+\cdots+f_{0}+f_{1} X^{-1 / M}+\cdots\right) .
\end{aligned}
$$

PROOF. The case $P_{0}=0$ being trivial, we may assume without loss of generality that $P_{0}>0$. Using Lemmas (2.2) and (2.6) we can write

$$
P_{0} \cdot P_{1}^{Q_{1}} \cdots \cdots P_{k}^{Q_{k}}=F_{\sigma} \cdot\left(e^{r} \cdot 2^{r_{1}} \cdots \cdots p_{n}^{r_{n}} \cdot X^{r_{\infty}}\right) \cdot\left(1+c_{1} X^{-1}+\cdots\right)
$$

for some tuple $\sigma$ and rational numbers $r_{0}, r_{1}, \ldots, r_{n}, r_{\infty}$, and a sequence of rationals $c_{1}, c_{2}, \ldots$

The last two factors on the right-hand side can be contracted in an obvious way to a fractional power series of the desired form.

We can now prove the result stated in the Introduction.

(2.11) THEOREM. Given any Skolem monomials $M_{1}, \ldots, M_{s}$ one can determine effectively whether $M_{1}+\cdots+M_{s}>0$, or $M_{1}+\cdots+M_{s}=0$, or $M_{1}+\cdots+M_{s}<0$.

ProOF. Write each $M_{i}$ as

$$
M_{i}=F_{\sigma(i)} \cdot\left(f_{-m i} X^{m / M}+F_{(-m+1) i} X^{(m-1) / M}+\cdots\right),
$$

according to the previous lemma. We can do this in such a way that all tuples $\sigma(i)$ are of the same length and the numbers $m, M$ are also independent of $i$. Using (2.8) and (2.9) we may also assume that, for some $t \in\{1, \ldots, s\}$, we have 
$\sigma(1)=\cdots=\sigma(t)=\sigma$, and $F_{\sigma(i)}(x) / F_{\sigma}(x)<e^{-c x}$ for $i=t+1, \ldots, s$, a constant $c>0$, and all sufficiently large $x$.

This implies that $M_{1}+\cdots+M_{t}$ dominates $M_{t+1}+\cdots+M_{s}$ in absolute value, provided $M_{1}+\cdots+M_{t} \neq 0$. We can effectively verify whether or not $M_{1}+\cdots+M_{t}=$ 0 by $[\mathbf{R 2}$ or $\mathbf{M}]$. If $M_{1}+\cdots+M_{t}=0$ we have a reduction to a sum of fewer Skolem monomials.

Suppose that $M_{1}+\cdots+M_{t} \neq 0$. Then we can effectively write

$$
M_{1}+\cdots+M_{t}=F_{\sigma} \cdot\left(g_{-m} X^{m / M}+g_{-m+1} X^{(m-1) / M}+\cdots+g_{0}+g_{1} X^{-1 / M}+\cdots\right) \text {, }
$$

where each coefficient $g_{j}$ is of the form $g_{j}=b_{i j} e^{r_{1 j}}+\cdots+b_{t j} e^{r_{t j}}$, with the $b_{i j}$ algebraic real numbers and the $r_{i j}$ rational numbers.

Since $e$ is transcendental we can find the first nonzero coefficient $g_{j}$ and determine its sign. If the sign is 1 , then $M_{1}+\cdots+M_{s}>0$, if the sign is -1 , then $M_{1}+\cdots+$ $M_{s}<0$.

\section{A correction.}

(3.1) In this section we assume sorne familiarity with the author's article [VdD]. At the end of that paper I claim, without proof, two (equivalent, but false) statements, $(*)$ and $(* *)$ (cf. [VdD, p. 65]).

Statement $(*)$ is as follows:

"For each nonzero $E$-polynomial $p(X)$ over $\left(\mathbf{R}, e^{x}\right)$, all $a, b \in \mathbf{R}$ with $a<b$, and each ordered $E$-extension field $F$ of $\left(\mathbf{R}, e^{x}\right)$ we have: all roots of $p(X)$ in $F$ between $a$ and $b$ are in $\mathbf{R}$." (Here, and in the following, an ordered $E$-field $F$ satisfies $E(x) \geq 1+x$, in accordance with the convention of [ VdD, p. 64].)

A counterexample to $(*)$ is implicit in $[\mathbf{D W}]$ : Take a positive infinitesimal $\alpha$ in some ordered field $R$ extending $\mathbf{R}$. As in the proof of Corollary 27 in [DW] we see that $(1+\alpha / n)^{n}<1+\alpha+\alpha^{2} / 2<(1-\alpha / n)^{n}$ for all natural numbers $n$, and therefore, according to Theorem 26 of $[\mathbf{D W}]$ we have $E(\alpha)=1+\alpha+\alpha^{2} / 2$ for a suitable ordered $E$-extension field $F$ of $\left(\mathbf{R}, e^{x}\right)$. But $E(X)-\left(1+X+X^{2} / 2\right)$ is an example of an $E$-polynomial over $\left(\mathbf{R}, e^{x}\right)$ which has no real root strictly between 0 and 1 , and we have a contradiction with $(*)$.

(3.2) The intention behind the (refuted) statement $(*)$ was to indicate universal axioms satisfied by $\left(\mathbf{R}, e^{x}\right)$ which, together with the axioms for $E$-fields and the diagram of $\left(\mathbf{R}, e^{x}\right)$, could prove every true instance of " $p(X)$ has exactly $k$ zeros between $a$ and $b$ " $\left(p(X) \in \mathbf{R}[X]^{E}, k \in \mathbf{N}, a, b \in \mathbf{R}\right)$. This intention can still be realized, but we need more than just the axiom $\forall x(E(x) \geq 1+x)$.

Let us define a nicely ordered $E$-field to be an ordered $E$-field $F$ such that

$$
\left|E(x)-E_{k}(x)\right| \leq \frac{e^{|x|} \cdot|x|^{k+1}}{(k+1) !} \quad \text { for all } x \in F, k \in \mathbf{N}
$$

where $E_{k}(x)=\sum_{i=0}^{k} x^{i} / i !$

Now we have the following result which comes in place of $(*)$.

(3.3) Proposition. Let $F$ be a nicely ordered E-extension field of $\left(\mathbf{R}, e^{x}\right)$, and $p(X) \in \mathbf{R}[X]^{E}, p \neq 0$. Then each finite zero of $p(X)$ in $F$ is in $\mathbf{R}$. 
PROOF. By induction on the complexity of $p(X)$ one shows first that for each $a \in \mathbf{R}$ and $\dot{k} \in \mathbf{N}$ there is a positive real constant $C$ such that

$$
\left|p(a+x)-\sum_{i=0}^{k} \frac{p^{(i)}(a)}{i !} x^{i}\right| \leq C|x|^{k+1}
$$

for all infinitesimals $x$ in $F$.

Let $b \in F$ be a finite zero of $p(X)$ where $p \neq 0$. Then $b=a+x$ for some $a \in \mathbf{R}$ and infinitesimal $x$. Suppose now that $b \notin \mathbf{R}$. Then $x \neq 0$, and it will suffice to derive a contradiction from this. Since $p \neq 0$ we can take $k \in \mathbf{N}$ so large that the Taylor polynomial

$$
f(X)=\sum_{i=0}^{k} \frac{p^{(i)}(a)}{i !} X^{i}
$$

is nonzero. From $p(b)=0$ it follows that $|f(x)| \leq C|x|^{k+1}$ for some positive real number $c$, and this contradicts $\operatorname{deg} f \leq k$.

\section{REFERENCES}

[DW] B. I. Dahn and H. Wolter, On the theory of exponential fields, Z. Math. Logik Grundlag. Math. 29 (1983), 465-480.

[G] R. Gurevic, Transcendental numbers and eventual dominance of exponential functions, preprint.

[H] G. H. Hardy, Orders of infinity, Cambridge Univ. Press, New York, 1910.

[HR] C. W. Henson and L. A. Rubel, Some applications of Nevanlinna theory to mathematical logic: Identities of exponential functions, Trans. Amer. Math. Soc. 282 (1984), 1-32.

[L] H. Levitz, Calculation of an order type: An application of non-standard methods, $\mathrm{Z}$. Math. Logik Grundlag. Math. 28 (1982), 219-228.

[M] A. Macintyre, The laws of exponentiation, Model Theory and Arithmetic, Lecture Notes in Math., vol. 890, Springer-Verlag, Berlin and New York, 1981, pp. 185-197.

[R1] D. Richardson, Some undecidable problems involving elementary functions of a real variable, J. Symbolic Logic 33 (1968), 514-520.

[R2] _ Solution of the identity problem for integral exponential functions, Z. Math. Logik Grundlag. Math. 15 (1969), 333-340.

[VdD] L. van den Dries, Exponential rings, exponential polynomials and exponential functions, Pacific J. Math. 113 (1984), 51-66. 94305

Department of Mathematics, Stanford University, Stanford, California

Current address: Max Planck Institute für Mathematik, Gottfried-Claren-Strasse 26, 5300 Bonn 3, Federal Republic of Germany 